\title{
Periodic leg movements in Cheyne-Stokes respiration
}

\author{
M. Manconi*, G. Vitale ${ }^{\#}$, R. Ferri`, M. Zucconi* and L. Ferini-Strambi*
}

ABSTRACT: Periodic leg movements during sleep (PLMS) are repetitive jerks of the lower limbs. Their occurrence can be isolated or part of various sleep disorders, such as restless legs syndrome (RLS), narcolepsy and obstructive sleep apnoea. Dopamine agonists are very effective in suppressing PLMS in RLS from the first night of administration. PLMS are often associated with cortical and autonomic arousals and may contribute to sleep disruption.

Herein, a case of association between PLMS, Cheyne-Stokes respiration (CSR) and severe insomnia is described.

Repeated polysomnographic studies demonstrated a clear synchronisation between PLMS and hyperventilation episodes, and the complete recovery of CSR, sleep quality and PLMS during nocturnal ventilation. In the same patient, pharmacological treatment with a dopamine agonist was ineffective on both PLMS and CSR.

These results suggest that periodic leg movements during sleep coupled with central breathing events, similar to those observed in conjunction with obstructive events, may have a different origin in terms of neurotransmitter and pathway implicated, compared with periodic leg movements during sleep in restless legs syndrome, and are resolved by treatment of the respiratory abnormalities.

KEYWORDS: Central sleep apnoea, Cheyne-Stokes respiration, polysomnography, sleep, sleep-disordered breathing

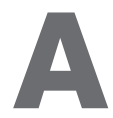
ccording to the recently revised scoring criteria, periodic leg movements during sleep (PLMS) are characterised by tibialis anterior electromyographic bursts ranging $0.5-$ $10 \mathrm{~s}$ in duration, with an amplitude increasing $>8 \mu \mathrm{V}$ above the resting voltage, separated by intermovement intervals of 5-90 s and occurring in series of at least four events [1]. PLMS are usually associated with cortical arousals, insomnia and excessive daytime sleepiness, but their pathogenetic role in sleep disruption is still debated [2].

Although the aetiopathogenesis of PLMS is unclear, several lines of evidence suggest a dopaminergic system dysfunction as their basic mechanism [3, 4]. In particular, low doses of dopamine agonists have been demonstrated to be extremely effective in suppressing PLMS from the first night of administration [5, 6]. PLMS mostly occur in patients with restless legs syndrome (RLS), but they are commonly observed also in other sleep disorders such as rapid eye movement (REM) sleep behaviour disorder or narcolepsy [7]. However, PLMS can be found in healthy subjects, especially in the elderly. Obstructive sleep apnoea (OSA) is a well established condition associated with PLMS [8]. In this case, PLMS typically occur synchronously with breathing resumption at the end of each apnoea, and are often suppressed by continuous positive airway pressure (CPAP). The close link between PLMS and apnoea, together with the responsiveness to $\mathrm{CPAP}$, suggest a different pared with PLMS in other sleep disorders. Indeed, the current scoring criteria establish that leg movements should not be scored as PLMS if they occur during a period of $0.5 \mathrm{~s}$ preceding or following an apnoea or hypopnoea [1]. The same concept might also be valid for sleep central breathing disorders, although this has not been investigated extensively. A higher prevalence of PLMS was found in patients with congestive heart failure than in normal controls, without any apparent correlation with the presence of pathogenesis of leg movements in OSA com-

\section{AFFILIATIONS}

${ }^{*}$ Centre of Sleep Medicine, Dept of Neurology, Scientific Institute of San Raffaele, Vita-Salute University, Milan,

\#Dept of Neurosciences, University of Catania, Catania, and

- Sleep Research Centre, Department of Neurology IC, Oasi Institute (IRCCS), Troina, Italy.

CORRESPONDENCE

M. Manconi

Sleep Disorders Center

Dept of Neurology

Vita-Salute University

IRCCS H S.Raffaele

Via Stamira d'Ancona 20

20127 Milan

Italy

Fax: 390226433394

E-mail: manconi.mauro@hsr.it

Received:

December 042007

Accepted after revision:

May 132008

STATEMENT OF INTEREST

None declared. 
Cheyne-Stokes respiration (CSR) [9]. Herein, a case of CSR with PLMS is reported, and the relationship between the two phenomena is investigated by observing their differential response to a dopamine agonist and to CPAP.

\section{CASE REPORT}

A 62-yr-old Caucasian male nonsmoker, who usually drank one small cup of coffee per day, was referred to the sleep centre because of his severe chronic insomnia, which started $\sim 2$ yrs before the current authors' evaluation. He exhibited no response to the common hypnotic medications. The patient was affected by a stable congestive heart failure due to ischaemic heart disease. The patient had undergone an aortocoronary bypass grafting 8 yrs previously, and a mechanic aortic valve replacement 2 yrs previously. No other significant diseases, such as metabolic or respiratory syndromes, were present in the medical history. At the moment of evaluation he was mildly overweight (body mass index $27.3 \mathrm{~kg} \cdot \mathrm{m}^{-2}$ ) and dyslipidaemic (cholesterol $266 \mathrm{mg} \cdot \mathrm{dL}^{-1}$, triglycerides $232 \mathrm{mg} \cdot \mathrm{dL}^{-1}$ ), with the following blood gas analysis values: oxygen tension $99.4 \mathrm{mmHg}$, carbon dioxide tension $36.1 \mathrm{mmHg}, \mathrm{pH} 7.39$ and arterial oxygen saturation $96.9 \%$. Serum iron storage indicators were within the normal range (ferritin $116 \mathrm{ng} \cdot \mathrm{mL}^{-1}$, iron $98 \mu \mathrm{g} \cdot \mathrm{dL}^{-1}$, transferrin $220 \mathrm{mg} \cdot \mathrm{dL}^{-1}$ ). The results of spirometry were unremarkable. The patient was taking oral anticoagulant therapy (international normalised ratio 1.9, prothrombin time $26.5 \mathrm{~s}$ ) and atorvastatin $\left(10 \mathrm{mg} \cdot \mathrm{day}^{-1}\right)$. No antidopaminergic or other types of drugs that could induce PLMS were taken. An echocardiographic study showed mild aortic dilatation $(43 \mathrm{~mm})$, mild concentric left ventricular hypertrophy, left ventricular ejection fraction of $55 \%$, mitral fibrosis with mild insufficiency and pulmonary pressure of $36 \mathrm{mmHg}$.

The patient complained of poor sleep, which was described as fragmented, agitated, not restoring and with a mean duration of 3-4 h.night ${ }^{-1}$. Daytime fatigue, somnolence and depression were also reported. No restless legs symptoms or other specific sleep disorders were reported. The patient underwent three nonconsecutive full-night polysomnographic (PSG) studies, each one preceded by an adaptation night in a standard soundattenuated sleep laboratory room. The following signals were recorded: electroencephalogram (EEG; six to 12 channels, from bipolar derivations), electrooculogram (electrodes placed $1 \mathrm{~cm}$ above the right outer canthus and $1 \mathrm{~cm}$ below the left outer canthus and referred to the mastoid), electromyogram (EMG) of the submentalis muscle, ECG, oronasal airflow (thermistor), thoracic and abdominal effort (strain gauge), EMG of the right and left tibialis anterior muscles (bipolar derivations with two electrodes placed $2 \mathrm{~cm}$ apart on the anterior tibialis muscle of each leg, with impedance $<10 \mathrm{k} \Omega$ ), and arterial oxygen saturation (pulse oximetry).

\section{PSG 1 (baseline night)}

The first baseline PSG showed a series of central apnoeas (apnoea/hypopnoea index (AHI) $35.6 \mathrm{~h}^{-1}$ ), associated with significant fluctuations in peripheral arterial oxygen saturation

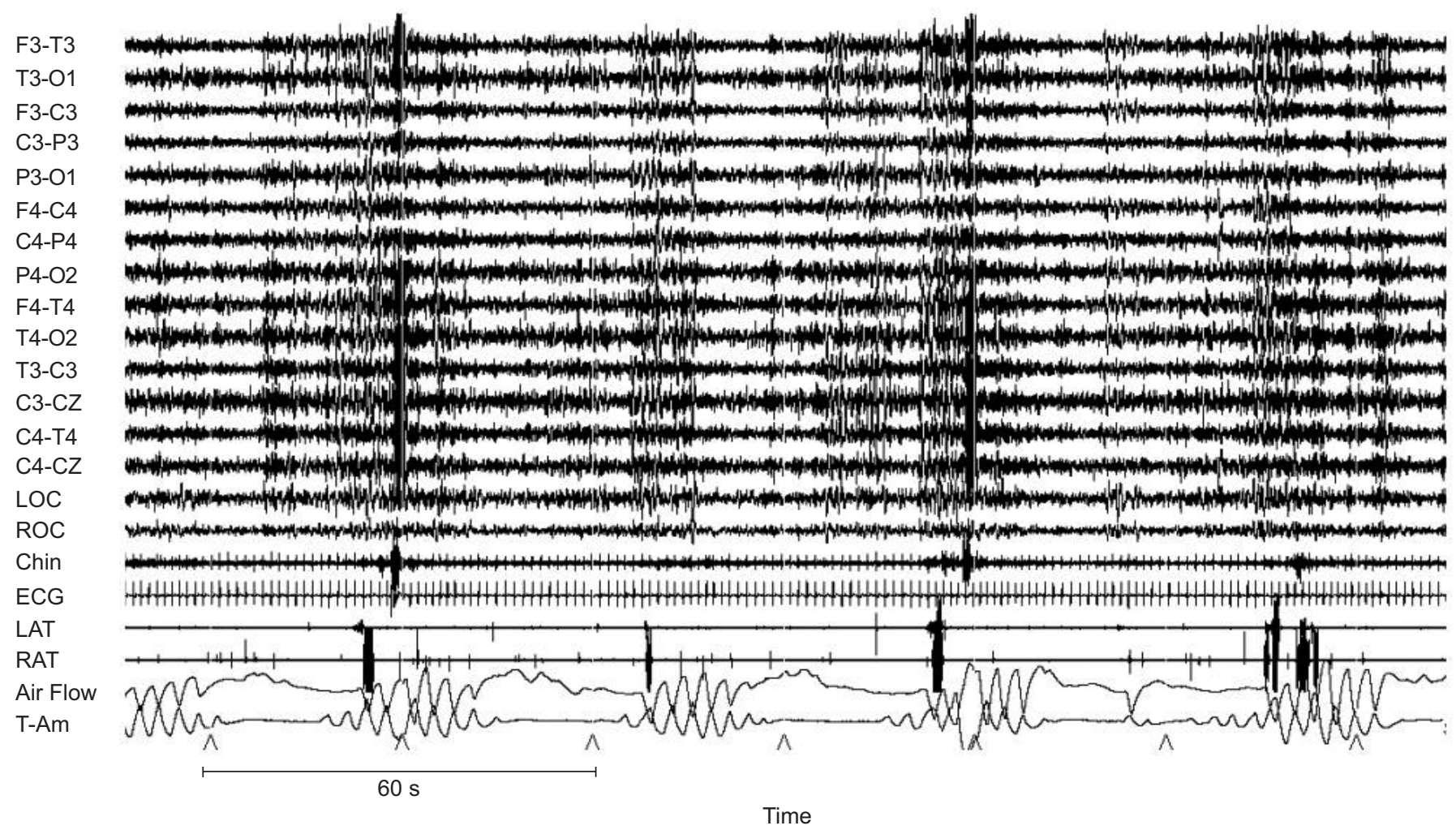

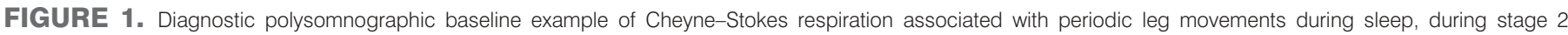

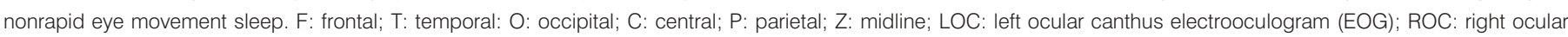
canthus EOG; Chin: submentalis muscle electromyogram; LAT: left tibialis anterior muscle; RAT: right tibialis anterior muscle; T-Am: thoraco-abdominal movements. 
(oxygen desaturation index $32.1 \mathrm{~h}^{-1}$ ) in the context of a CSR pattern. Breathing events were prevalent in non-REM (NREM) sleep (fig. 1), especially during sleep stages 1 and 2 . They were rare in slow-wave sleep and disappeared in REM sleep. The lowest arterial oxygen saturation was $79 \%$ and the percentage of time spent with arterial oxygen saturation $<90 \%$ was $10 \%$. No snoring or obstructive events were detected. Periodic respiration was usually associated with cortical arousals, increases in cardiac frequency $(f C)$ and bilateral tibialis anterior muscle EMG bursts, all occurring during the maximum amplitude of the breathing oscillation. On the basis of the first 20 periodic respiratory events, the following mean $\pm \mathrm{SD}$ values were calculated: mean \pm SD apnoea duration $27.3 \pm 4.8$ s; hyperpnoea duration $18.9 \pm 4.7 \mathrm{~s}$; respiratory rate during the hyperpnoea $21.1 \pm 1.8 \mathrm{~s}^{-1}$; and circulation time $11.8 \pm 2.3 \mathrm{~s}$. There was no clear correlation between the level of hypoxaemia and the above-mentioned parameters with the arousal-related events. The synchronisation between cortical arousals, leg movements and the point of arousal or of highest breathing amplitude was constant across the night. No PLMS were recorded during airflow flattening and during periods free from CSR. Sleep architecture was disrupted, with significant reductions in sleep efficiency $(57 \%)$, total sleep time $(236 \mathrm{~min})$ and percentage of slow-wave $(11.2 \%)$ and REM sleep $(9.9 \%)$. According to the standard diagnostic criteria, the tibialis anterior EMG bursts belonged to the PLMS category, the PLMS index (number of periodic leg movements per hour of sleep) was $34.1 \mathrm{~h}^{-1}$, and the PLMS arousal index (number of periodic leg movements associated with an EEG arousal per hour of sleep) was $33.2 \mathrm{~h}^{-1}$. An arousal was defined as an abrupt shift of EEG frequency including alpha, theta and/or frequencies $>16 \mathrm{~Hz}$ (but not spindles), during NREM sleep, that lasts $\geqslant 3 \mathrm{~s}$, with $\geqslant 10 \mathrm{~s}$ of stable sleep preceding the change. An arousal event and movement event are considered associated with each other when there is $<0.5 \mathrm{~s}$ between the end of one event and the onset of the other event, regardless of which is first $[1,10]$.

A detailed neurophysiological study of EEG and $f C$ changes accompanying PLMS was also carried out. In order to reduce or eliminate baseline variability of the parameters considered, the current authors used the same methodology that was successfully applied in a previous study on these measures in patients with RLS [11], a fixed-time window of $50 \mathrm{~s}(20 \mathrm{~s}$ preceding and $30 \mathrm{~s}$ following the onset of each PLMS) was used, with the first $10 \mathrm{~s}$ serving for the calculation of the baseline; subsequently, each value was expressed as a percentage of this baseline value, for all parameters calculated around each PLMS (10 PLMS from NREM sleep were selected randomly for this analysis). $f(C$ was measured and its value calculated for each round second by means of a linear interpolation between the measured values; EEG power spectrum was calculated for frequencies between $0.5-30 \mathrm{~Hz}$ from the central (C)4-temporal (T)4 derivation, by means of a 2-s sliding window, which was moved along the 50-s epoch with steps of $1 \mathrm{~s}$. Subsequently, the power spectrum for the following frequency bands was obtained for each time step (delta: $0.5-2.5 \mathrm{~Hz}$; theta: 4.0-7.5 Hz; alpha: 7.5-11.0 Hz; and beta: $15.00-30.0 \mathrm{~Hz}$ ). In this way, a normalised synchronous graph was obtained of $f C$ and of the different EEG bands, which also allowed us to characterise the time relationships between them.
Figure 2 shows the timing of the changes in the different parameters considered. The delta and the theta bands show an increase preceding the PLMS onset by some seconds and a sustained decrease after the PLMS, while the beta band shows an increase which follows the PLMS onset by some seconds; finally, the alpha band shows a minor sustained decrease after the PLMS. $f C$ shows a clear and sustained increase starting with the onset of the PLMS.

\section{PSG 2 (nocturnal ventilation night)}

During the second PSG study, a nasal mask CPAP, with oxygen supplementation $\left(1-2 \mathrm{~L} \cdot \mathrm{min}^{-1}\right)$, was performed. With a pressure of $5 \mathrm{cmH}_{2} \mathrm{O}$, both central apnoeas (AHI $1.7 \mathrm{~h}^{-1}$ ) and PLMS (PLMS index $1.2 \mathrm{~h}^{-1}$ ) recovered completely during nocturnal ventilation (fig. 3). Arterial oxygen saturation was always $>90 \%$, with a lowest point at $94 \%$. As shown in table 1 , a considerable improvement in sleep architecture parameters was also evident during CPAP treatment.

\section{PSG 3 (dopamine-agonist medication night)}

The patient underwent a third PSG study without CPAP. Before this study, a single dose of pramipexole $0.25 \mathrm{mg}$ was administered to the patient at 21:00 h. Sleep (sleep efficiency $67.8 \%$ ), breathing (AHI $32.1 \mathrm{~h}^{-1}$ ) and leg movement (PLMS index $20.2 \mathrm{~h}^{-1}$, PLMS arousal index $19.6 \mathrm{~h}^{-1}$ ) parameters were almost unaffected by drug medication, and were roughly comparable to those recorded during the baseline PSG (fig. 4). The lowest arterial oxygen saturation was $80 \%$ and the percentage of time with arterial oxygen saturation $<90 \%$ was $9 \%$.

Chronic treatment with CPAP $\left(5 \mathrm{cmH}_{2} \mathrm{O}\right.$ with $2 \mathrm{~L} \cdot \mathrm{min}^{-1}$ of oxygen supplementation) was prescribed and, after a 1-yr follow up, a PSG study confirmed the good tolerance and the efficacy of nocturnal ventilation in controlling both breathing and movement events. After a further 7 months, and after 2 days of CPAP discontinuation, an additional basal PSG study, by means of a nasal pressure cannula instead of the thermistor, was performed. The same CSR pattern (AHI was $29.2 \mathrm{~h}^{-1}$, PLMS index was $26.8 \mathrm{~h}^{-1}$, lowest arterial oxygen saturation was $81 \%$, percentage of time with arterial oxygen saturation $<90 \%$ was $9 \%$ ), together with the absence of an upper airway obstructive component, was found.

\section{DISCUSSION}

In the present study, the association between CSR and PLMS has been documented by repeated PSG studies. The same association has been reported in the literature only by HANLY and ZUBERI [12], who observed a patient affected by congestive heart failure, insomnia, CSR and PLMS. In that case [12], PLMS were partially related to CSR, occurring not only during the hyperpnoeic phases but also during regular breathing and during resting wakefulness, and they disappeared together with the CSR after heart transplantation. Beyond the association between CSR and PLMS, the case herein reported and the case described by HANLY and ZUBERI [12], present further common features such as sleep disruption and recovery of PLMS after CSR resolution. However, in this case, as shown in figures 1 and 4, PLMS were strictly correlated with the last portion of the crescendo of the hyperpnoeic respiratory phases, tachycardia and cortical arousals, and they never occurred during apnoea or outside of CSR. In both cases, the presence of severe insomnia, which improved immediately after the 

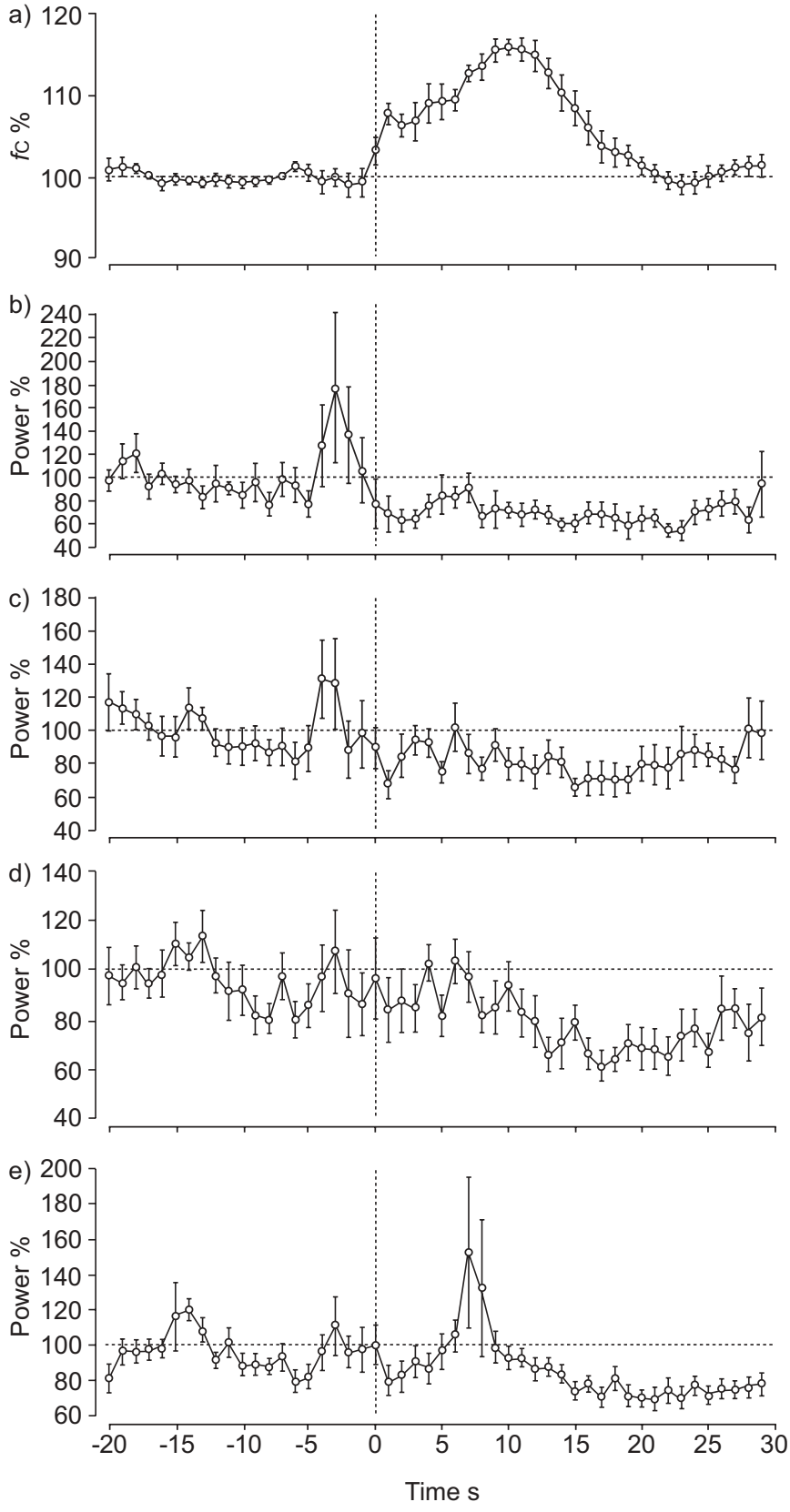

FIGURE 2. Changes in a) cardiac frequency $(f c)$ and $b-e)$ the power of different electroencephalogram bands (b: delta $(0.5-2.5 \mathrm{~Hz})$; c: theta $(4.0-7.5 \mathrm{~Hz})$; d: alpha $(7.5-11.0 \mathrm{~Hz})$; e: beta $(15.0-30.0 \mathrm{~Hz})$ ) accompanying periodic leg movements during nonrapid eye movement sleep on polysomnography night 1 (baseline night). Data are shown as a percentage of the average value calculated over the first $10 \mathrm{~s}$ of a 50-s window, from 20-10 min before the onset of leg movements (dashed vertical line).

disappearance of PLMS and CSR, suggests a possible role of leg movements in association with the breathing abnormalities, in inducing sleep disruption. Periodic and nonperiodic leg movements during sleep are well known to be related with significant EEG, fC [11] and blood pressure changes [13], with a possible impact on sleep quality. Moreover, a higher cardiac activation has been observed when leg movements are coupled with the end of OSA, compared with respiratory events without leg movements [14]. GUGGISBERG et al. [15] reported, in normal subjects, that $f C$ changes associated with sleep-related leg movement preceded leg movements and were greater when occurring with periodic leg movements than with either isolated or respiratory-related leg movements. In the view of GUGGISBERG et al. [15], their results might suggest a primary role of the sympathetic nervous system in the generation of PLMS. Since CPAP reduces sympathetic activity in patients with sleep apnoea, the recovery of PLM by CPAP might support the hypothesis of GUGGISBERG et al. [15]. However, other studies performed in patients with RLS [11, 13, 16] or sleep apnoea [14] have reported essentially opposite findings. For these reasons, it is difficult to interpret the findings of the current study as an effect of increased sympathetic activity, even if this cannot be completely excluded.

The synchronisation among cortical, motor and autonomic phenomena recalls the so-called cyclic alternating pattern (CAP), which is a spontaneous or stimulus-induced oscillation in brain activity occurring with a periodicity of 20-40 s [17]. Composed by an activation component (phase A), followed by a deactivation component (phase B), CAP fluctuations modulate and interact with the time structure of physiological and pathological events. CAP oscillations are particularly stereotyped in OSA, where respiration is interrupted during CAP phase $B$ and restored during phase A [18]. Although related to a central and nonobstructive breathing disorder, the same trend also seems to be followed in the present case, where PLMS occur during CAP phase $\mathrm{A}$ and never during the inhibitory phase $\mathrm{B}$, as if their time structure is under a common central control. In the case described herein, the EEG slow components usually preceded the motor events, which in turn were followed by rapid EEG oscillations. The first slow EEG oscillations are very likely to be part of phase A of CAP, while the beta peak may be the perturbation effect on EEG activity induced by the motor and sympathetic activation.

To the current authors' knowledge, this is the first documentation of the efficacy of CPAP in eliminating PLMS associated with CSR. Furthermore, the authors tried to treat the PLMS/ CSR association with pramipexole but found no effect. The PLMS response to a higher dose of pramipexole may be different from what was observed in this case; however, the clearly different results obtained after two separate therapeutic approaches suggest two important speculations: 1) CSR may play an active role in inducing PLMS; and 2) these PLMS might not be primarily dependent on dopaminergic dysfunction. The diagnostic criteria for PLMS [1] identify a spectrum of leg movements with an apparently common PSG phenotype, which may include motor events with a different pathogenesis in terms of neurotransmitter or nervous pathways implicated in their generation. Recently, the investigation of the time structure of leg movements by means of a new analysis [19], has enabled detection of considerable differences in several parameters, such as periodicity, duration, and sleep stage distribution, between PLMS in RLS [20] and those associated with narcolepsy [21] or with REM sleep behaviour disorder [22]. These differences are not detectable by means of the simple conventional PLMS scoring method and corroborate the idea of a possible pathogenetic heterogeneity in the phenomenon generically defined as PLMS. 


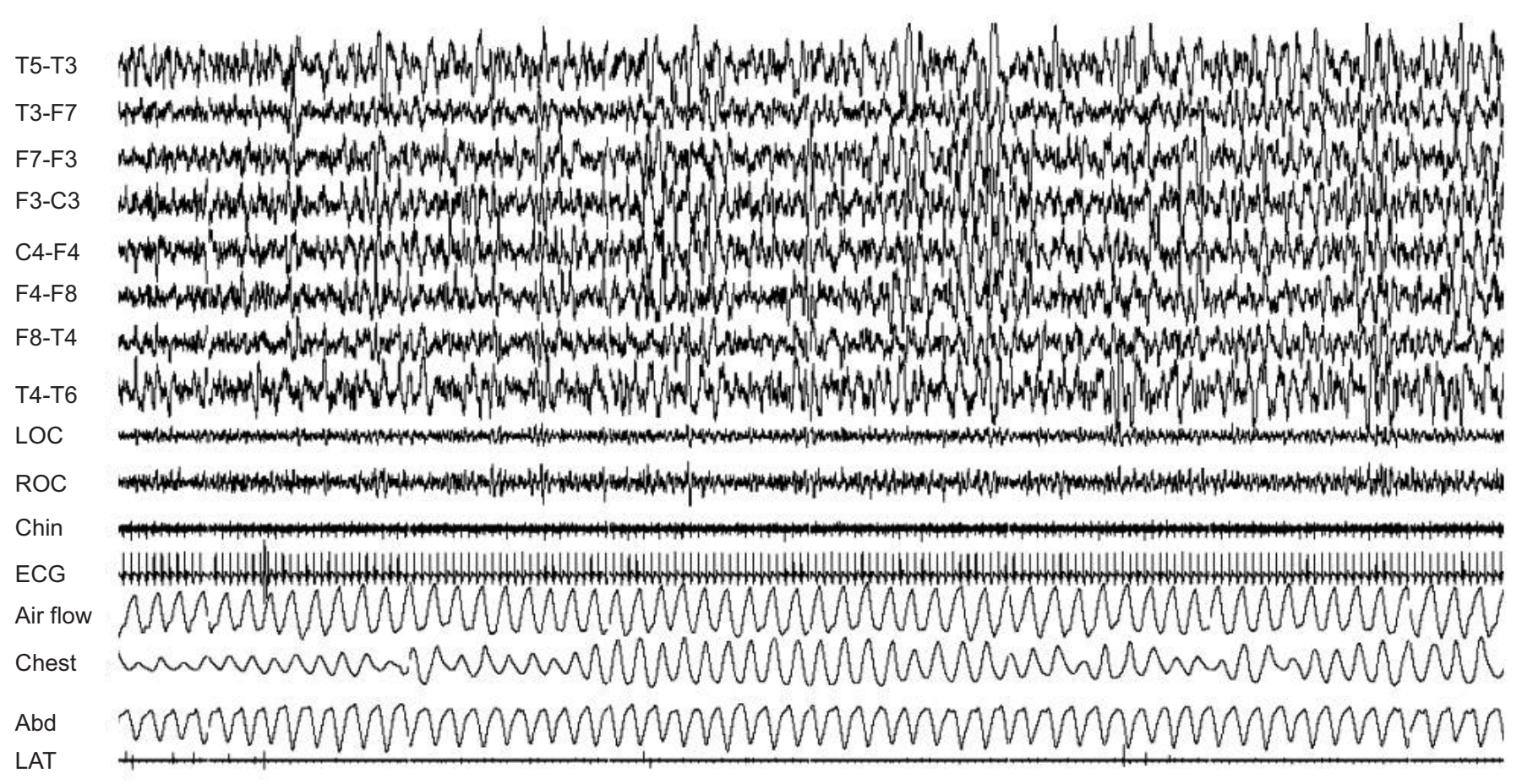

$60 \mathrm{~s}$

Time

FIGURE 3. Polysomnographic example (stage 4 nonrapid eye movement sleep) during continuous positive airway pressure application. There is an absence of CheyneStokes respiration and periodic leg movements during sleep. T: temporal; F: frontal; C: central; LOC: left ocular canthus electrooculogram (EOG); ROC: right ocular canthus EOG; Chin: submentalis muscle electromyogram; Abd: abdominal movements, LAT: left tibialis anterior muscle; RAT: right tibialis anterior muscle.

\begin{tabular}{|c|c|c|c|}
\hline \multirow[t]{2}{*}{ TABLE 1} & \multicolumn{3}{|c|}{$\begin{array}{l}\text { Results of the three polysomnographic studies } \\
\text { performed in baseline condition, during } \\
\text { nocturnal ventilation and during pramipexole } \\
\text { treatment }\end{array}$} \\
\hline & Baseline & CPAP & Pramipexole \\
\hline TST min & 236 & 351 & 264 \\
\hline SEI \% & 56.9 & 83.7 & 67.8 \\
\hline WASO min & 159 & 53 & 101 \\
\hline SL min & 20 & 15 & 26 \\
\hline NREM $S_{1}-S_{2} \%$ & 78.8 & 71.1 & 59.7 \\
\hline NREM $S_{3}-S_{4} \%$ & 10.1 & 17.2 & 20.4 \\
\hline REM sleep \% & 9.9 & 11.5 & 19.8 \\
\hline AHI $h^{-1}$ & 35.6 & 1.7 & 32.1 \\
\hline ODI $h^{-1}$ & 32.1 & 1.2 & 30.6 \\
\hline PLMS index $h^{-1}$ & 34.1 & 0 & 20.2 \\
\hline
\end{tabular}

Contrary to PLMS associated with OSA, where leg movements occurring $0.5 \mathrm{~s}$ preceding or following the end of a breathing episode are not scored [1], herein the time which separated the end of the apnoea/hypopnoea from the motor event always exceeded $0.5 \mathrm{~s}$, and this tibialis anterior activity should be classified as PLMS. Since, in both OSA and CSR, leg movements are synchronised with the end of apnoeas, and are usually recovered by CPAP [10], perhaps too leg movements related to CSR should not be scored as typical PLMS. Further studies on the differential effect of pharmacological versus ventilation therapy are warranted in order to understand the nature of CSR-associated PLMS.

In conclusion, the current authors recommend the inclusion of the recording of tibialis anterior muscles in polysomnographic studies of patients with Cheyne-Stokes respiration, especially if they present sleep complaints. At the same time, the current authors strongly suggest that the breathing pattern of patients with periodic leg movements during sleep should be studied always, with the aim of avoiding the use of unhelpful and potentially unsafe dopamine agonists in subjects with CheyneStokes respiration (because of their well-known cardiac side effects), in favour of the specific continuous positive airway pressure treatment. 


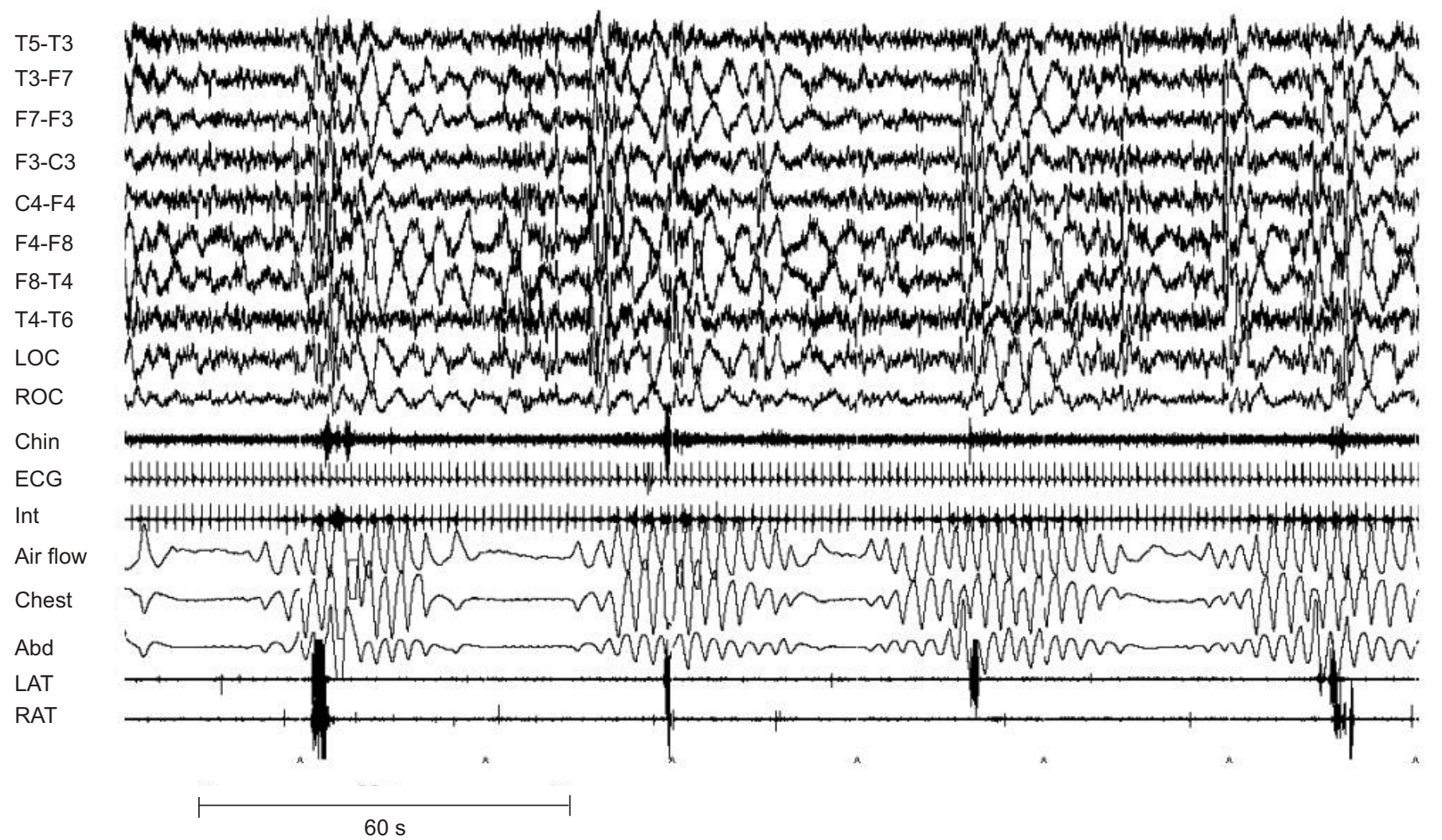

Time

FIGURE 4. Polysomnographic example (stage 2 nonrapid eye movement sleep) after pramipexole treatment with the reoccurrence of Cheyne-Stokes respiration and periodic leg movements during sleep. T: temporal; F: frontal; C: central; LOC: left ocular canthus electrooculogram (EOG); ROC: right ocular canthus EOG; Chin: submentalis muscle electromyogram; Int: intercostal muscle; Abd: abdominal movements, LAT: left tibialis anterior muscle; RAT: right tibialis anterior muscle.

\section{ACKNOWLEDGEMENTS}

The current authors would like thank V. Patruno (Hospital Unit of Respiratory Rehabilitation, Ospedale S. Marta, Ospedale Maggiore di Crema (CR), Rivolta D'adda, Italy) for his contribution to the analysis of polysomnographic respiratory data.

\section{REFERENCES}

1 Iber C, Ancoli-Israel S, Chesson A Jr, Quan SF, eds, The AASM Manual for the Scoring of Sleep and Associated Events. Rules, Terminology and Technical Specifications. 1st Edn. Westchester, American Academy of Sleep Medicine, 2007.

2 Nicolas A, Lesperance P, Montplaisir J. Is excessive daytime sleepiness with periodic leg movements during sleep a specific diagnostic category? Eur Neurol 1998; 40: 22-26.

3 Clemens S, Rye D, Hochman S. Restless legs syndrome: revisiting the dopamine hypothesis from the spinal cord perspective. Neurology 2006; 67: 125-130.

4 Montplaisir J, Lorrain D, Godbout R. Restless legs syndrome and periodic leg movements in sleep: the primary role of dopaminergic mechanism. Eur Neurol 1991; 31: 41-43.

5 Manconi M, Ferri R, Zucconi M, et al. First night efficacy of pramipexole in restless legs syndrome and periodic leg movements. Sleep Med 2007; 8: 491-497.
6 Saletu M, Anderer P, Saletu B, et al. Sleep laboratory studies in restless legs syndrome patients as compared with normals and acute effects of ropinirole. 2. Findings on periodic leg movements, arousals and respiratory variables. Neuropsychobiology 2000; 41: 190-199.

7 Hornyak M, Feige B, Riemann D, Voderholzer U. Periodic leg movements in sleep and periodic limb movement disorder: prevalence, clinical significance and treatment. Sleep Med Rev 2006; 10: 169-177.

8 Warnes H, Dinner DS, Kotagal P, Burgess RC. Periodic limb movements and sleep apnoea. J Sleep Res 1993; 2: 38-44.

9 Hanly PJ, Zuberi-Khokhar N. Periodic limb movements during sleep in patients with congestive heart failure. Chest 1996; 109: 1497-1502.

10 Zucconi M, Ferri R, Allen R, et al. The official World Association of Sleep Medicine (WASM) standards for recording and scoring periodic leg movements in sleep (PLMS) and wakefulness (PLMW) developed in collaboration with a task force from the International Restless Legs Syndrome Study Group (IRLSSG). Sleep Med 2006; 7: 175-183.

11 Ferri R, Zucconi M, Rundo F, Spruyt K, Manconi M, FeriniStrambi L. Heart rate and spectral EEG changes accompanying periodic and non-periodic leg movements during sleep. Clin Neurophysiol 2007; 118: 438-448. 
12 Hanly P, Zuberi N. Periodic leg movements during sleep before and after heart transplantation. Sleep 1992; 15: 489-492.

13 Pennestri MH, Montplaisir J, Colombo R, Lavigne G, Lanfranchi PA. Nocturnal blood pressure changes in patients with restless legs syndrome. Neurology 2007; 68: 1213-1218.

14 Yang CK, Jordan AS, White DP, Winkelman JW. Heart rate response to respiratory events with or without leg movements. Sleep 2006; 29: 553-556.

15 Guggisberg AG, Hess CW, Mathis J. The significance of the sympathetic nervous system in the pathophysiology of periodic leg movements in sleep. Sleep 2007; 30: 755-766.

16 Ferrillo F, Beelke M, Canovaro P, et al. Changes in cerebral and autonomic activity heralding periodic limb movements in sleep. Sleep Med 2004; 5: 407-412.

17 Terzano MG, Parrino L. Origin and significance of the cyclic alternating pattern (CAP). Sleep Med Rev 2000; 4: 101-123.

18 Thomas RJ, Terzano MG, Parrino L, Weiss JW. Obstructive sleep-disordered breathing with a dominant cyclic alternating pattern - a recognizable polysomnographic variant with practical clinical implications. Sleep 2004; 27: 229-234.

19 Ferri R, Zucconi M, Manconi M, et al. Computer-assisted detection of nocturnal leg motor activity in patients with restless legs syndrome and periodic leg movements during sleep. Sleep 2005; 28: 998-1004.

20 Ferri R, Zucconi M, Manconi M, Plazzi G, Bruni O, FeriniStrambi L. New approaches to the study of periodic leg movements during sleep in restless legs syndrome. Sleep 2006; 29: 759-769.

21 Ferri R, Zucconi M, Manconi M, et al. Different periodicity and time structure of leg movements during sleep in narcolepsy/cataplexy and restless legs syndrome. Sleep 2006; 29: 1587-1594.

22 Manconi M, Ferri R, Zucconi M, Fantini L, Plazzi G, FeriniStrambi L. Time structure analysis of leg movements during sleep in REM sleep behavior disorder. Sleep 2007; 30: 1779-1785. 